\title{
COMMENT
}

\section{Understanding positive child health}

Jacqueline T. Bangma $\mathbb{D}^{1}$, Evan Kwiatkowski ${ }^{2}$, Matt Psioda ${ }^{2}$, Hudson P. Santos $\mathrm{Jr}^{3}$, Stephen R. Hooper ${ }^{4}$, Laurie Douglass ${ }^{5}$, Robert M. Joseph ${ }^{6}$, Jean A. Frazier ${ }^{7}$, Karl C. K. Kuban ${ }^{8}$, Thomas M. O'Shea ${ }^{9}$ and Rebecca C. Fry' ${ }^{1}$, for the ELGAN Investigators

Pediatric Research (2019) 86:690-691; https://doi.org/10.1038/s41390-019-0564-8

In response to the commentary, ${ }^{1}$ we would like to thank the authors for their interest and thoughtful comments.

As a first step toward addressing limitations of measures of child health, Bangma et al. ${ }^{2}$ shows that the positive child health index $(\mathrm{PCHI})$ is correlated with the Pediatric Quality of Life Inventory (PedsQL), which includes measures of quality of life across physical and psychosocial domains as reported by a parent or guardian. The developed PCHI aims to make a connection on the state of well-being of the child beyond the perception of a parent or guardian. Agreement between the two measures suggests they could be both making inferences on a more complete notion of positive health.

Once determined to be associated with the PedQL, the strength of Bangma et al. ${ }^{2}$ was the development of a PCHI measure that utilizes disease susceptibility or health to further interrogate aspects of positive child health. There are, however, aspects of positive health that can only be ascertained from the individual directly. In the ongoing evaluation of the ELGAN cohort at 15 years of age, the team is broadening the assessment of positive aspects of health, with measurement of adolescent-reported quality of life, life satisfaction, meaning and purpose, and global health, as well as measures of sleep quality. These assessments will provide aspects of well-being that were not possible to obtain at the 10year evaluation, and, in conjunction with the $\mathrm{PCHl}$, will provide the necessary components for a truly comprehensive measure of positive heath.

A primary goal of Bangma et al. $^{3}$ on antecedents of $\mathrm{PCHI}$ was to begin the search for factors associated with increased likelihood of positive outcomes that might be targeted as treatments/intervention strategies for all children. It is clear that some of the antecedents are likely to be more easily modifiable than others and thus should be prioritized for future intervention trials.

There are important long-term implications for programs, practices, and policies that could promote positive child health, particularly in relation to children born prematurely. The current research collaborations, made possible by the Environmental Influences on Child Health Outcomes (ECHO) Program, will be enhanced by the opportunity to engage in interdisciplinary collaborations that involve experts in medical and behavioral interventions as well as experts in social determinants of health.
Finally, all research is designed to stimulate additional dialogue about not only the merits of the science but also the advancement of scientific inquiry into a particular topic. We are pleased to see that this paper has begun an important discussion into the myriad of issues inherent in positive child health.

\section{ACKNOWLEDGEMENTS}

This study was supported by grants from the National Institute of Neurological Disorders and Stroke (5U01NS040069-05; 2R01NS040069-06A2), the National Institute of Child Health and Human Development (5R01HD092374-02 and 5P30HD01865534), the Office of the NIH Director (1UG3OD023348-01), NIH training grant (T32ES007018), and National Institute of Nursing Research (1K23NR017898-01). The authors also gratefully acknowledge the contributions of the ELGAN subjects, the ELGAN subjects' families, as well as colleagues listed below:

\section{Site Principal Investigators}

Baystate Medical Center, Springfield, MA: Bhahvesh Shah, MD; Rachana Singh, MD, MS. Boston Children's Hospital, Boston, MA: Linda Van Marter, MD, MPH; Camilla Martin, MD, MPH; Janice Ware, PhD. Tufts Medical Center, Boston, MA: Cynthia Cole, MD; Ellen Perrin, MD. University of Massachusetts Medical School, Worcester, MA: Frank Bednarek, MD; Jean A. Frazier, MD. Yale University School of Medicine, New Haven, CT: Richard Ehrenkranz, MD; Jennifer Benjamin, MD. Wake Forest University, Winston-Salem, NC: T. Michael O'Shea, MD, MPH. University of North Carolina, Chapel Hill, NC: Carl Bose, MD; Diane Warner, MD, MPH. East Carolina University, Greenville, NC: Steve Engelke, MD. Helen DeVos Children's Hospital, Grand Rapids, MI: Mariel Poortenga, MD; Steve Pastyrnak, PhD. Sparrow Hospital, East Lansing, MI: Padu Karna, MD; Nigel Paneth, MD, MPH; Madeleine Lenski, MPH. University of Chicago Medical Center, Chicago, IL: Michael Schreiber, MD; Scott Hunter, PhD; Michael Msall, MC. William Beaumont Hospital, Royal Oak, MI: Danny Batton, MD; Judith Klarr, MD.

\section{Site Study Coordinators}

Baystate Medical Center, Springfield, MA: Karen Christianson, RN; Deborah Klein, BSM, RN. Boston Children's Hospital, Boston, MA: Maureen Pimental, BA; Collen Hallisey, BA; Taryn Coster, BA. Tufts Medical Center, Boston, MA: Ellen Nylen, RN; Emily Neger, MA; Kathryn Mattern, BA. University of Massachusetts Medical School, Worcester, MA: Lauren Venuti, BA; Beth Powers, RN; Ann Foley, EdM. Yale University School of Medicine, New Haven, CT: Joanne Williams, RN; Elaine Romano, APRN. Wake Forest University, Winston-Salem, NC: Debbie Hiatt, BSN (deceased); Nancy Peters, RN; Patricia Brown, RN; Emily Ansusinha, BA. University of North Carolina, Chapel Hill, NC: Gennie Bose, RN; Janice Wereszczak, MSN; Janice Bernhardt, MS, RN. East Carolina University, Greenville, NC: Joan Adams (deceased); Donna Wilson, BA, BSW. Nancy Darden-Saad, BS, RN. Helen DeVos Children's Hospital, Grand Rapids, MI: Dinah Sutton, RN; Julie Rathbun, BSW, BSN. Sparrow Hospital, East Lansing, MI: Karen Miras, RN, BSN; Deborah Weiland, MSN. University of Chicago Medical Center, Chicago, IL:

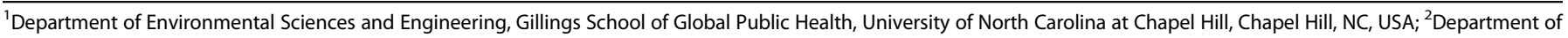

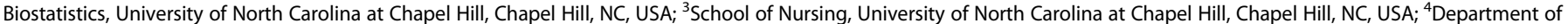

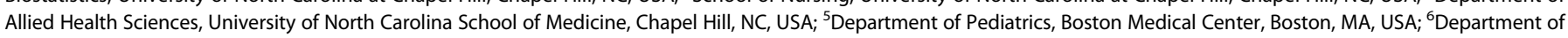

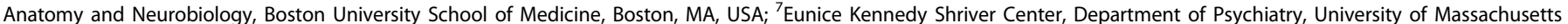

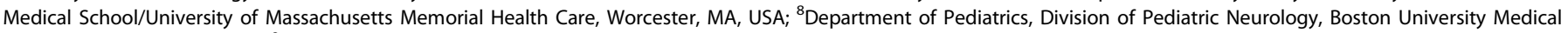
Center, Boston, MA, USA and ${ }^{9}$ Department of Pediatrics, University of North Carolina, Chapel Hill, NC, USA

Correspondence: Jacqueline T. Bangma (jbangma@ad.unc.edu)

These authors contributed equally: Jacqueline T. Bangma, Evan Kwiatkowski
}

Received: 29 June 2019 Accepted: 17 August 2019

Published online: 14 September 2019 
Grace Yoon, RN; Rugile Ramoskaite, BA; Suzanne Wiggins, MA; Krissy Washington, MA; Ryan Martin, MA; Barbara Prendergast, BSN, RN. William Beaumont Hospital, Royal Oak, MI: Beth Kring, RN.

\section{Psychologists}

Baystate Medical Center, Springfield, MA: Anne Smith, PhD; Susan McQuiston, PhD. Boston Children's Hospital: Samantha Butler, PhD; Rachel Wilson, PhD; Kirsten McGhee, PhD; Patricia Lee, PhD; Aimee Asgarian, PhD; Anjali Sadhwani, PhD; Brandi Henson, PsyD. Tufts Medical Center, Boston, MA: Cecelia Keller, PT, MHA; Jenifer Walkowiak, PhD; Susan Barron, PhD. University of Massachusetts Medical School, Worcester MA: Alice Miller, PT, MS; Brian Dessureau, PhD; Molly Wood, PhD; Jill Damon-Minow, PhD. Yale University School of Medicine, New Haven, CT: Elaine Romano, MSN; Linda Mayes, PhD; Kathy Tsatsanis, PhD; Katarzyna Chawarska, PhD; Sophy Kim, PhD; Susan Dieterich, PhD; Karen Bearrs, PhD. Wake Forest University Baptist Medical Center, Winston-Salem NC: Ellen Waldrep, MA; Jackie Friedman, PhD; Gail Hounshell, PhD; Debbie Allred, PhD. University Health Systems of Eastern Carolina, Greenville, NC: Rebecca Helms, PhD; Lynn Whitley, PhD Gary Stainback, PhD. University of North Carolina at Chapel Hill, NC: Lisa Bostic, OTR/L; Amanda Jacobson, PT; Joni McKeeman, PhD; Echo Meyer, PhD. Helen DeVos Children's Hospital, Grand Rapids, Ml: Steve Pastyrnak, PhD. Sparrow Hospital, Lansing, Ml: Joan Price, EdS Megan Lloyd, MA, EdS. University of Chicago Medical Center, Chicago, IL: Susan Plesha-Troyke, OT; Megan Scott, PhD. William Beaumont Hospital, Royal Oak, MI: Katherine M. Solomon, PhD; Kara Brooklier, PhD; Kelly Vogt, PhD.

\section{AUTHOR CONTRIBUTIONS}

\section{ADDITIONAL INFORMATION}

Competing interests: The authors declare no competing interests.

Publisher's note Springer Nature remains neutral with regard to jurisdictional claims in published maps and institutional affiliations.

\section{REFERENCES}

1. Litt, J. S. and H. Tiemeier. Positive health: rebranding an old package with a new name? Pediatr. Res. https://doi.org/10.1038/s41390-019-0454-0 (2019). [Epub ahead of print].

2. Bangma, J. T., et al. Assessing positive child health among individuals born extremely preterm. J. Pediatr. 202, 44-49 (2018).

3. Bangma, J. T., et al. Early life antecedents of positive child health among 10-yearold children born extremely preterm. Pediatr. Res. https://www.nature.com/ articles/s41390-019-0404-x (2019). [Epub ahead of print]. 